\title{
BMJ Open Prevalence and factors associated with medication adherence among patients with hypertension in sub-Saharan Africa: protocol for a systematic review and meta-analysis
}

Valirie Ndip Agbor, ${ }^{1}$ Noah Fongwen Takah, ${ }^{2,3}$ Leopold Ndemnge Aminde ${ }^{4,5}$

To cite: Agbor VN, Takah NF, Aminde LN. Prevalence and factors associated with medication adherence among patients with hypertension in sub-Saharan Africa: protocol for a systematic review and meta-analysis. BMJ Open 2018;8:e020715. doi:10.1136/ bmjopen-2017-020715

- Prepublication history and additional material for this paper are available online. To view these files, please visit the journal online (http://dx.doi. org/10.1136/bmjopen-2017020715).

Received 19 November 2017 Revised 20 January 2018 Accepted 31 January 2018

Check for updates

${ }^{1}$ Ibal Sub-Divisional Hospital, Oku, North west, Cameroon ${ }^{2}$ Ministry of Public Health, Yaounde, Cameroon

${ }^{3}$ London School of Hygiene and Tropical Medicine, London, UK ${ }^{4}$ Non-Communicable Disease Unit, Clinical Research

Education, Networking and Consultancy (CRENC), Douala, Cameroon

${ }^{5}$ School of Public Health, Faculty of Medicine, The University of Queensland, Brisbane, Queensland, Australia

Correspondence to Dr Leopold Ndemnge Aminde; amindeln@gmail.com

\section{ABSTRACT}

Introduction Hypertension is the leading cardiovascular risk factor globally, associated with a high morbidity and mortality. The high prevalence of hypertension in subSaharan Africa (SSA) is associated with contrastingly low awareness, treatment and control rates. Adherence to medication remains a major determinant of optimal blood pressure control. This systematic review aims to determine the prevalence, and factors associated with adherence to antihypertensive pharmacotherapy among patients with hypertension in SSA.

Methods and analysis We will include studies published in Africa up to 31 December 2017. The following databases will be searched: PubMed, Embase, SCOPUS and Web of Science to identify potential studies without language restriction. To minimise chances of missing studies, resources specific to African literature such as WHO AFROLIB, African Index Medicus and African Journals Online will also be searched. Two reviewers will independently screen studies, extract data and critically appraise included studies for risk of bias, and a third reviewer will resolve discrepancies. A random-effects meta-analysis is planned to pool study-specific estimates to obtain a summary measure presented in Forest plots. Heterogeneity of included studies will be assessed using the $\chi^{2}$ test on Cochrane's $Q$ statistic and quantified using $\mathrm{I}^{2}$. Publication bias will be assessed using the Egger's test and funnel plots. This protocol has been prepared according to the Preferred Reporting Items for Systematic Reviews and Metaanalyses for Protocols 2015 statement.

Ethical and dissemination An ethical approval is not required for the proposed study, as it will be based on already published data. The end report will be presented at conferences and published in a peer-reviewed journal. PROSPERO registration number CRD42017079838.

\section{INTRODUCTION}

Hypertension is the principal risk factor for cardiovascular diseases (CVD), whose sequelae are associated with significant morbidity and mortality worldwide. Globally, hypertension accounts for about nine million
Strengths and limitations of this study

- To the best of the authors' knowledge, this will be the first comprehensive systematic review and meta-analysis on the prevalence and predictors of adherence to antihypertensive pharmacotherapy among patients with hypertension in sub-Saharan Africa (SSA).

- We anticipate that with the largely sensitive search strategy, this systematic review will capture the maximum available studies reporting on the prevalence and predictors of adherence to antihypertensive medication, and the results will be disaggregated according to the different SSA regions and type of method or tool used to assess medication adherence.

- A limitation of this study might be the absence of or limited number of community-based studies on the subject.

- There is currently no universally agreed method and/or tool used to evaluate medication adherence. Additionally, the use of arbitrary cut-off values to sometimes define adherence remains a potential source of substantial heterogeneity we anticipate, while assessing the prevalence of adherence to antihypertensive pharmacotherapy reported across studies done on the subject in SSA.

deaths among the one billion adults living with the condition. ${ }^{12}$ Sub-Saharan Africa (SSA) has experienced a steady increase in the prevalence of hypertension from $9.7 \%$ to $30.8 \%$ in 1990 and 2010 , respectively, ${ }^{3}$ with remarkable regional variations ranging from $15 \%$ to $70 \% .^{2-5}$ This high prevalence of hypertension on the continent is associated with contrastingly low awareness, treatment and control rates. ${ }^{3}$

Adherence, as defined by the WHO, is 'the extent to which a person's behaviourtaking medication, following a diet and/or executing lifestyle changes-corresponds 
with agreed recommendations from a healthcare provider'. ${ }^{6}$ Non-adherence to pharmacological therapy, especially in patients with chronic health conditions, is a growing concern worldwide which constitutes a major barrier to safe, cost-effective and effective use of drugs. ${ }^{6}$ The factors driving patients' adherence to medication are multifactorial, but can be grouped under five main domains ${ }^{7}$ including socioeconomic factors such as health literacy, medication cost, availability of health insurance, cultural beliefs about the illness and treatment. Second, factors related to the healthcare system such as providerpatient relationship, provider communication skills, presence of community care and short waiting time. Third, factors related to conditions such as absence of symptoms, chronic conditions, depression and psychotic disorders. Fourth, therapy-related factors such as duration of therapy, frequency of changes in medication, absence of side effects, number of daily doses and absence of concurrent medication and finally, patient-related factors such as motivation, good knowledge about medication and perceived benefit of treatment. ${ }^{7}$ Adherence to diet and lifestyle modifications, and antihypertensive pharmacotherapy are pivotal in achieving optimal blood pressure (BP) control, especially for patients whose BP cannot be controlled by diet and lifestyle modification alone. Congruently, an optimal BP is crucial in the prevention of complications such as heart failure, stroke, ischaemic heart disease, kidney disease and hypertension-related mortality. ${ }^{8-10}$ Thus, improving adherence to antihypertensive pharmacotherapy is key to ameliorating outcomes in patients with hypertension. In addition, understanding the determinants of adherence to antihypertensive pharmacotherapy among patients living with hypertension in SSA is crucial in guiding policy-makers to tailor effective strategies to improve patient adherence to antihypertensive pharmacotherapy, and consequently BP control.

In a recent review by Abegaz et al, ${ }^{11} 45.3 \%$ of patients with hypertension globally were non-adherent to their antihypertensive medication, with a non-adherence rate as high as $62.5 \%$ reported among African patients on treatment for hypertension. However, it is very likely that their prevalence estimate of non-adherence to antihypertensive medications might not capture the full picture of adherence in Africa for a number of reasons. First, this study had a relatively narrow window of study inclusion. Second, they restricted their inclusion to studies assessing medication adherence using a single tool. Third, their search was limited to only two databases without any search of the existing African-specific data sources. Importantly, a rigorous critical appraisal of the quality of included studies for this review was not done. As such, the quality of evidence from this review seems wanting, coupled with the low sensitivity of their search strategy, demonstrated in the very limited number of included studies from Africa. Drivers of this medication compliance problem were similarly not explored. Taken together, their findings present significant shortcomings limiting its ability to capture a comprehensive picture of antihypertensive medication adherence in Africa.

Considering the above caveats and compelling need for comprehensive context-specific evidence to address the burden of hypertension and CVD, we decided to conduct this systematic review and meta-analysis with focus on SSA. Our aim is to determine the prevalence of adherence to antihypertensive pharmacotherapy among patients with hypertension in SSA and to investigate the factors associated with medication adherence, which have hitherto not been comprehensively synthesised in SSA.

\section{Objective}

The aim of this systematic review and meta-analysis is to determine the prevalence of adherence to antihypertensive pharmacotherapy and the factors associated with medication adherence among patients with hypertension in SSA.

\section{Review question}

This review aims to answer the following questions:

1. What is the current prevalence of adherence to antihypertensive pharmacotherapy among patients with hypertension in SSA?

2. What are the factors associated with adherence to antihypertensive pharmacotherapy among patients with hypertension in SSA?

\section{METHODS AND ANALYSIS}

\section{Criteria for considering studies for the review}

Inclusion criteria

Studies reporting on human subjects with the criteria mentioned below will be considered for inclusion.

1. Observational studies with available data on the prevalence (cross-sectional, case series with at least 30 participants and cohort studies) and factors associated with adherence to antihypertensive pharmacotherapy (cross-sectional, case-control, cohort and randomised control trials) among patients with hypertension residing in SSA.

2. Participants of at least 18 years of age residing in SSA.

3. Publication date: all published and unpublished literature up to 31 December 2017 will be considered for the review, without any language restriction.

\section{Exclusion criteria}

The following studies will not be considered:

1. Studies conducted among participants of non-SSA origin or of SSA origin but residing outside the region.

2. Editorials, letters to the editor, commentaries and case series with less than 30 participants.

3. Studies without relevant data to compute the prevalence or predictors of adherence to antihypertensive pharmacotherapy among patients with hypertension.

4. Duplicate studies: here, the most comprehensive and/or recent study with the largest sample size will be considered. 
5. Studies with incomplete data, even after request from the corresponding author.

\section{SOURCE OF INFORMATION}

\section{Search strategy for identifying relevant studies}

Data sources and search strategy

Using medical subject headings $(\mathrm{MeSH})$ and key text words, such as 'adherence', 'compliance', 'medication', 'drug' or 'antihypertensive', the abstracts of published articles with relevant information on the prevalence and/ or factors associated with adherence to antihypertensive pharmacotherapy among patients with hypertension in SSA will be identified. This will be achieved through a search of databases including PubMed, Embase, SCOPUS and Web of Science from inception to 31 December 2017. To minimise chances of missing relevant studies, resources specific to African literature such as WHO AFROLIB, African Index Medicus and African Journals Online will also be searched. To improve the sensitivity and precision of our search geographically, we shall use a validated search filter proposed for Africa. ${ }^{12}$ The main search strategy for PubMed is displayed in table 1.

Thereafter, potential abstracts will be reviewed and their full texts retrieved through Embase, PubMed, Scopus, Google scholar, Sci Hub or the journal's website. After retrieving the full texts of relevant articles and reviews on the subject, their reference lists will be examined to identify other relevant articles not captured during our search. The full texts of these articles will be retrieved as described above.

\section{Grey literature}

Google Scholar will be used to search for grey literature. We shall also search conference websites and those of hypertension and cardiology societies in Africa such as the Pan-African Society of Cardiology for relevant material. Where full text of an article cannot be accessed from any of the aforementioned sources, the corresponding author will be contacted via email or other platforms such as ResearchGate. In the absence of a response from the authors after multiple attempts, the said study will be excluded.

\section{Study records}

Data management

The studies obtained from the database searches shall be imported to EndNote V.7.4 software for removal of duplicates. The remaining articles will be uploaded to Rayyan QCRI ${ }^{13}$ which is a web-based and mobile application that facilitates collaboration between reviewers involved in the screening and selection of studies to be finally included in a systematic review. A tool shall be developed a priori according to eligibility criteria to guide the process of study selection.

\section{Screening}

The titles and abstracts of papers retrieved from the search will be carefully screened, and the full text of potentially eligible articles retrieved as earlier discussed. This exercise will be conducted independently by two authors (VNA and NFT), who will further review the full texts of potential articles for final inclusion. The authors will compare their results at every step of the selection process, and discrepancies will be resolved through discussion and consensus. A third author (LNA) will be consulted in case of any disagreement. In the event of unclear or ambiguous information, the corresponding author of the said study shall be contacted for clarification.

\section{Data items and extraction}

Two reviewers (VNA and NFT) shall independently extract data from the full texts of included studies using a predefined data extraction sheet. Any disagreements or inconsistencies shall be resolved by consensus or consultation with third reviewer (LNA). Data will be extracted according to the outcome of interest: (1) prevalence and (2) factors associated with adherence to antihypertensive pharmacotherapy.

\section{Prevalence of adherence to antihypertensive pharmacotherapy}

The following items shall be extracted: the last name of the first author and year of publication, year(s) the study was conducted, the country and region (Southern, Eastern, Western and Central Africa) where the study was conducted, study design, study setting (rural vs urban) and study type (community based vs hospital based). Data on method used to assess medication adherence including self-report (using tools such as Brief Medication Questionnaire, Eight-Item Morisky Medication Adherence Scale and Medication Adherence Report Scale), or pill count, electronic monitoring, pharmacy records and prescription claims, biological assay will be collected. The approach to data collection (face-toface interview vs self-administered), sample size, total number of cases adherent to antihypertensive medication, mean or median doses taken, mean or median age and age range in years and the male proportion in the respective studies will also be obtained.

The three different components of adherence: initiation (the time interval from prescription of antihypertensive medication until when the patient took the first dose of his medication), implementation (the time from initiation to when the patient took the last dose of his antihypertensive medications) and discontinuation (the point when the patient misses the next dose to his treatment and no further dose is taken, this marks the end of therapy) will be considered when defining adherence to antihypertensive pharmacotherapy. ${ }^{14}$

\section{Factors associated with adherence to antihypertensive}

\section{pharmacotherapy}

In addition to general data items obtained in the previous section, the measure of association (OR or relative risk with their respective CIs) for each associated factor will be extracted and specification made if 


\section{SN Search items}

1. 'Medication Adherence'[Mesh] AND 'Patient Compliance'[Mesh] OR adherence or compliance OR (predictor OR 'associat` factor' OR determinant)

2. Antihypertensive Agents'[Mesh] OR antihypertensive* or 'blood pressure lowering' or 'antihypertensive pharmacotherapy'

3. \#1 AND \#2

4. benin/or burkina faso/or cape verde/or cote d'ivoire/or gambia/or ghana/or guinea/or guinea-bissau/or liberia/or mali/ or mauritania/or nigeria/or senegal/or sierra leone/or togo/or ((africa*adj2 west* or benin* or burkina fas ${ }^{\star}$ or cape verd $^{\star}$ or cabo verd* or ivory coast or cote d'ivoire* or gambia* or ghana* or (guinea* not pig*) or bissau or liberia* or (mali not fowl) or malian or mauritania* or nigeria* or senegal* or sierra leon* or togo*).mp. or (Lagos or Accra or Abidjan or Dakar or Abobo or Abuja or Freetown or Ouagadougou or Conakry or Lome or Bamako or Cotonou or Kumasi or Monrovia or Ibadan or Kano or Port harcourt or Benin City or Porto Novo or Niamey or Yamoussoukro or Banjul or Timbuktu or Djenne or Abomeyu or Zaria or Tamale or Jos or Cape Coast or Maidugul or Aba or Gao or Calabar or Warri or Maiduguri or Bobo Dioulasso or Parakou or Djougou or Bohicon or Sekondi Takoradi or Sunyani or Obuasi or Teshie or Tema or Sikasso or Kalabankoro or Nouakchott or Dakhlet Nouadhibou or Benin City or Port Harcourt or Ilorin or Kaduna or Enugu or Ikorodu or Onitsha or Bauchi or Akure or Abeokuta or Sokoto or Bouake or Makeni or Kaduan or Sosgbo or Osogbo or Gombe or llesa or Badagry or makurdi or Sagamu or Iseyin or obbomosho or Awka or Ado Ekiti or Nsukka or Ikeja or Katsina or Okene or Lafia or Minna or Ondo city or Umuahia or Calabar or Yola or Pikine or Touba or Thies Nones or Saint Louis or Kolak or Ziguinch or (San Pedro not (Spain or Mexico or Argentina or California or United States or Italy)) or Bandama or Daloa or Owerri or Kandi or Ifi or Dakar or Ogbomosho or Divo or Korhogo)).ti,ab or Exp africa, central/or ((africa adj2 central) or angola or cameroon* or chad.mp. or tchad.mp. or congo* or DRC or equatorial guinea* or gabon* or Sao Tome or Principe or Luanda or lobito or kuito or huambo or Malanje or Douala or Yaounde or Bamenda or Garoua of Bafoussam or Nganoundere or Maroua or Kouosseri or Buena or Kumba or N'Djamena or Moundou or Bangui or Bimbo or Brazzaville or Point Noire or Kinshasa or Lubumbashi or Leopoldville or Elizabethville or Mbuji Mayi or Bakwanga or Bukavu or Costermansville or Kananga or Luluabourg or Kisangani or Stanleyville or Tshikapa or Koalwezi or Likasi or Jadotville or Goma or Kikwit or Uvira or Bunia or Mbandaka or Coquilhatville or Matadi or Butembo or Kabinda or Mwene Ditu or Isiro or Paulis or Boma or Kindu or Bata or Malabo or Libreville).ti,ab or Exp Africa, Eastern/or ((east* adj2 africa*) or British Indian Ocean Territory or Burundi* or Comoros or Djibouti* or Eritrea* or Ethiopia* or Kenya* or Madagascar or Malawi or Mauritius or Mayotte or Mozambique or Reunion OR Rwanda* or Seychelles or Somalia* or Sudan* or Tanzania* or Uganda* or Zambia or Zimbabwe or Crozet Islands or lles Crozet or Scattered Islands or lles Eparses or Mwanza or Zanzibar or Eldoret or Morogoro or Hargeysa or Berbera or Nyeri or Mbeya or Machakos or Marka or Tabora or Iringa or Gondar or Meru or Geita or Musoma or Mtwara or Songea or Kigoma or Dese or Mek'ele or Bahir Dar or Jimma or Sinyanga or Korogwe or Nairobi or 'Dar es Salaam' or Mombasa or Addis Ababa or Kampala or Kigali or Mogadishu or Dodomoa or Bujumbura or Nakuru or Anananarivo or Kisumu or Maputo or Asmara or Lusaka or Harare or Port Louis or Arusha or kitale or lilongwe or malindi or machakos or hargeisa or Bulawayo or Ruiru or Lamu or Kire Dawa or Kikuyu or naivasha or mwanza or tanga or nanyuki or voi or garissa or lodwar of kakamega or maralal or kitui or webuye or Axum or Nyahururu or Jinja or Kismayo or Namanga or Mumias or Moshi or Moroni or Lokichogio or Hola or Rwenzori Mountains or Lake Victoria or Puntland* or (Adiharush or Ali-Addeh or Alinjugur or Buramino or Dadaab or Dagahaley or Dollo Ado or Fugnido or Hagadera or Hilaweyn or Ifo or Kakuma or Kambioos or Kayaka II or Kobe or Kyangwali Nakivale or Nyarugusu or Wad Sherife or Bokolmanyo or Melkadida or Rwamanja) adj5 (camp or refug*)).ti,ab or angola/or botswana/or lesotho/ or malawi/or mozambique/or namibia/or south africa/or swaziland/or zambia/or zimbabwe/or ((africa* adj2 south*) or

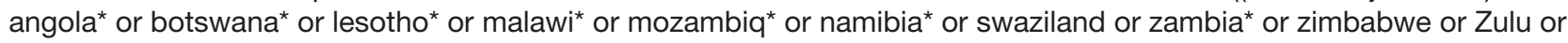
Tsonga or Xhosa or Swazi or Ndebele or Tswana or Sotho or Shona people or BaLunda or Mbundu or Ovimbundu or Chaga or Sukuma or Pretoria or Cape Town or Johannesburg or Durban or Port Elizabeth or Bloemfontein or Windhoek or Maseru or Pietermaritz or (Kimberley not Australia) or Nespruit or Soweto or Polokwane or Limpopo or Rustenburg or Mahikeng or Oudtshroom or Stellenbosch or Paarl or Gaborone or Luanda or Cabinda or Huambo or Lubango or Kuit or Malanje or Lobito or Lilongwe or Blantyre or Mzuzu or Maputo or Matola or Beira or Nampula or Chimoio or Nacala or Quelimane or Lusaka or Kitwe or Ndola or Kabwe or Copperbelt Harare or Bulawayo or Chitungwiza or Mutare or Masvingo or Monashonaland or Manicaland).ti,ab.

5. \#3 AND \#4

6. Publication date limits: from database inception to 31 December 2017, with no language restrictions

obtained from a univariate or multivariate analysis. In case of multivariate analysis, the variables adjusted for will also be obtained.

Overall, for multinational studies, the prevalence of adherence to hypertensive medication as well as the associated factors will be disaggregated and reported separately for individual studies. In case it is impossible to separate individual country data for a multinational study, we will present as a single study, and the individual countries in which the study was conducted in will be highlighted.

Assessment of methodological quality and risk of bias The risk of bias tool for prevalence studies developed by Hoy and colleagues ${ }^{15}$ will be used to assess the quality 
and risk of bias among included studies reporting on the prevalence of adherence to antihypertensive pharmacotherapy (see online supplementary file 1 for Hoy et al tool). On the other hand, the Quality In Prognosis Studies tool will be used to assess the risk of bias for cohort and prognostic studies reporting the factors associated with medication adherence. ${ }^{16}$ The risk of bias assessment will be conducted independently by two authors (VNA and NFT). The risk of bias for included studies will be presented in a tabular form.

\section{Data synthesis and analysis}

The Stata software V.15 will be used to analyse extracted data. The Cohen's $\kappa$ coefficient will be used to assess the inter-rater reliability for study inclusion. ${ }^{17}$

\section{Prevalence of adherence to antihypertensive pharmacotherapy}

A meta-analysis will be conducted for identical variables obtained from similar studies (studies using the same type of method/tool to assess adherence) to determine the pooled prevalence of adherence to antihypertensive pharmacotherapy. Assuming a binomial distribution, point estimates and appropriate denominators will be used to determine standard errors for study-specific estimates and the upper and lower bound of their CIs. Using a random-effect meta-analysis model, study-specific estimates will be pooled to determine the overall prevalence estimate across studies. The Freeman-Tukey double arcsine transformation will be used to stabilise the variance of the individual studies before pooling study-specific estimates. ${ }^{18}$ The Cochrane's $Q$ statistic ${ }^{19}$ and $\mathrm{I}^{2}$ values will be used to assess and quantify heterogeneity across studies, respectively. $\mathrm{I}^{2}$ values of $25 \%, 50 \%$ and $75 \%$ will represent low, medium and substantial heterogeneity, respectively ${ }^{20}$ In case of substantial clinical and/or methodological heterogeneity, we will perform a subgroup analysis using the following variables: age group (below vs at or above the median), sex (male vs female), comorbidities (presence vs absence) and duration of treatment (below vs at or above the median). Other variables that will be investigated include study design (cross-sectional vs cohort studies), study setting (rural vs urban), study type (hospital-based vs community-based), tool used to evaluate adherence, method used to assess medication adherence, method of data collection (face-to-face interview vs self-administered), geographical region (Southern, Eastern, Western and Central Africa) and study quality. A difference between subgroups will be considered significant if the $\mathrm{P}$ value is below 5\%. Finally, funnel plots and Egger's test will be used to assess the presence of publication bias. ${ }^{21} \mathrm{~A} \mathrm{P}$ value $<10 \%$ on Egger's test will be used to confirm the presence of publication bias.

\section{Factors associated with adherence to antihypertensive pharmacotherapy}

It is anticipated that there will be significant variation in factors associated with adherence to antihypertensive medication that have been investigated either clinically or methodologically/statistically. As such, pooling such data may put to question the reliability of the final summary estimate. Available data on these identified factors will thus be summarised in tabular form accompanied with a narrative discussion.

\section{Confidence in cumulative evidence}

The Grading of Recommendation Assessment Development and Evaluation approach will be used to evaluate the strength of evidence provided by the studies included in the final review by assessing the consistency, risk of bias and publication bias. Depending on whether further research is capable of changing the effect size, likely to have considerable impact on the effect size or unlikely to change the effect size, the studies will be described as 'low', 'moderate' and 'high' quality, respectively.

\section{Presentation and reporting of results}

The Preferred Reporting Items for Systematic Reviews and Meta-analyses (PRISMA) guidelines will be used to publish the proposed systematic review. ${ }^{22}$ The PRISMA checklist will also be published alongside the final review (online supplementary file 2). The entire process of study screening, selection and inclusion will be depicted with the aid of a flow diagram. Reasons for study exclusion will be documented and summary shown in the flow diagram. Where appropriate, quantitative data will be presented on forest plots and summary tables.

The prevalence of adherence to antihypertensive pharmacotherapy will be reported according to the study setting (hospital-based vs community-based study), tool (eg, Brief Medication Questionnaire, Eight-Item Morisky Medication Adherence Scale and Medication Adherence Report Scale) and method used to evaluate medication adherence. Additionally, tables and narrative summaries will be used to report the risk of bias for every eligible study.

\section{Protocol amendments}

We do not plan to amend this protocol. Nevertheless, in case of any modifications, these will be explicitly addressed in our final review report.

Contributors LNA conceived the study. VNA, NFT and LNA designed the study protocol. VNA drafted the initial manuscript. NFT and LNA critically revised the protocol for methodological and intellectual content. LNA is the guarantor of the review. All authors read and approved the final version of the manuscript prior to submission.

Funding This research received no specific grant from any funding agency in the public, commercial or not-for-profit sectors.

Competing interests None declared.

Patient consent Detail has been removed from this case description/these case descriptions to ensure anonymity. The editors and reviewers have seen the detailed information available and are satisfied that the information backs up the case the authors are making.

Provenance and peer review Not commissioned; externally peer reviewed.

Open Access This is an Open Access article distributed in accordance with the Creative Commons Attribution Non Commercial (CC BY-NC 4.0) license, which permits others to distribute, remix, adapt, build upon this work non-commercially, and license their derivative works on different terms, provided the original work is 
properly cited and the use is non-commercial. See: http://creativecommons.org/ licenses/by-nc/4.0/

(C) Article author(s) (or their employer(s) unless otherwise stated in the text of the article) 2018. All rights reserved. No commercial use is permitted unless otherwise expressly granted.

\section{REFERENCES}

1. Lim SS, Vos T, Flaxman AD, et al. A comparative risk assessment of burden of disease and injury attributable to 67 risk factors and risk factor clusters in 21 regions, 1990-2010: a systematic analysis for the Global Burden of Disease Study 2010. Lancet 2012;380:2224-60.

2. WHO. Global brief on hypertension. $-2017 \mathrm{http}: / /$ ish-world.com/ downloads/pdf/global_brief_hypertension.pdf

3. Adeloye D. An estimate of the incidence and prevalence of stroke in Africa: a systematic review and meta-analysis. PLoS One 2014;9:e100724.

4. Mills KT, Bundy JD, Kelly TN, et al. Global disparities of hypertension prevalence and control: a systematic analysis of population-based studies from 90 countries. Circulation 2016;134:441-50.

5. Ataklte F, Erqou S, Kaptoge S, et al. Burden of undiagnosed hypertension in sub-saharan Africa: a systematic review and metaanalysis. Hypertension 2015;65:291-8.

6. Sabaté E. ProjectWA to LTT, network GAI, diseases WHOD of M of N. Adherence tolong-term therapies : evidence for action. $2003 \mathrm{http}: / /$ www.who.int/iris/handle/10665/42682 (cited 25 Sep 2017).

7. Ferdinand $\mathrm{KC}$, Senatore FF, Clayton-Jeter $\mathrm{H}$, et al. Improving Medication Adherence in Cardiometabolic Disease. J Am Coll Cardiol 2017;69:437-51.

8. James PA, Oparil S, Carter BL, et al. 2014 evidence-based quideline for the management of high blood pressure in adults: report from the panel members appointed to the Eighth Joint National Committee (JNC 8). JAMA 2014;311:507-20.
9. Rosendorff C, Black HR, Cannon CP, et al. Treatment of hypertension in the prevention and management of ischemic heart disease: a scientific statement from the american heart association council for high blood pressure research and the councils on clinical cardiology and epidemiology and prevention. Circulation 2007;115:2761-88.

10. Špinar J. Hypertension and ischemic heart disease. Cor Vasa 2012;54:e433-e438.

11. Abegaz TM, Shehab A, Gebreyohannes EA, et al. Nonadherence to antihypertensive drugs. Medicine 2017;96:e5641.

12. Sandy C. Health sciences search filters: geographic filters for Africa. Edmonton, AB: University of Alberta, 2017.

13. Ouzzani M, Hammady H, Fedorowicz Z, et al. Rayyan-a web and mobile app for systematic reviews. Syst Rev 2016;5:210.

14. Vrijens B, De Geest S, Hughes DA, et al. A new taxonomy for describing and defining adherence to medications. $\mathrm{Br} \mathrm{J}$ Clin Pharmacol 2012;73:691-705.

15. Hoy D, Brooks $\mathrm{P}$, Woolf $\mathrm{A}$, et al. Assessing risk of bias in prevalence studies: modification of an existing tool and evidence of interrater agreement. J Clin Epidemiol 2012;65:934-9.

16. Hayden JA, van der Windt DA, Cartwright JL, et al. Assessing bias in studies of prognostic factors. Ann Intern Med 2013;158:280.

17. McHugh ML. Interrater reliability: the kappa statistic. Biochem Med 2012;22:276-82.

18. Miller JJ. The inverse of the freeman - tukey double arcsine transformation. The American Statistician 1978;32:138.

19. Cochran WG. The combination of estimates from different experiments. Biometrics 1954;10:101-29.

20. Higgins JP, Thompson SG. Quantifying heterogeneity in a metaanalysis. Stat Med 2002;21:1539-58.

21. Egger M, Davey Smith G, Schneider M, et al. Bias in meta-analysis detected by a simple, graphical test. BMJ 1997;315:629-34.

22. Moher D, Liberati A, Tetzlaff J, et al. Preferred reporting items for systematic reviews and meta-analyses: the PRISMA statement. PLoS Med 2009;6:e1000097. 